\title{
Label-Free Biodetection Using Capacitive Micromachined Ultrasonic Transducers (CMUTs) and Its Application for Cardiovascular Disease Diagnostics
}

\author{
Sarayoot Eaimkhong ${ }^{1,2}$, Matthias Steiert ${ }^{2,3}$, Theresa F. Harper ${ }^{3}$, Michael D. Cable ${ }^{2,3}$ and James Gimzewski ${ }^{1,2,4 *}$ \\ ${ }^{1}$ Chemistry and Biochemistry Department, University of California-Los Angeles, Los Angeles, California, USA \\ ${ }^{2}$ California NanoSystems Institute, University of California-Los Angeles, Los Angeles, California, USA \\ ${ }^{3}$ Matrix Sensor Inc., Pleasanton, California, USA \\ ${ }^{4}$ International Center for Materials Nanoarchitectonics Satellite (MANA), National Institute for Materials Science (NIMS), Tsukuba, Japan
}

\begin{abstract}
In this study, Capacitive Micromachined Ultrasonic Transducers (CMUTs) were used to detect protein using two different schemes involving antibody detection and biomarker or antigen detection. For antibody detection, anti-human IgG Fab-specific was used as an antibody that specifically adsorbs on human IgG functionalized CMUT devices and anti-goat IgG was chosen as a non-specific negative control. We achieved a significant higher signal for the specific antibodies ( 10-500 $\mathrm{ng} / \mathrm{ml})$ on CMUT devices in comparison to the non-specific negative control at low concentration. For antigen detection, we were interested in a cardiovascular disease biomarker and chose the tumor necrosis factor alpha (TNF- $\alpha$ ) as the analyte. We found a linear calibration curve for TNF- $\alpha$ at very low concentrations and demonstrated that this label-free biodetection has a general applicability for assays including diagnostics of cardiovascular disease.
\end{abstract}

Keywords: Label-free detection; Capacitive Micromachined Ultrasonic Transducers (CMUTs); Biosensors; Cardiovascular diseases; Biomarkers

\section{Introduction}

Capacitive Micromachined Ultrasonic Transducer (CMUT) is a technology that was introduced as an alternative to piezoelectric resonators in the last decade. Currently the most recent designs were developed to have a high resonant frequency i.e. 40-52 $\mathrm{MHz}$. The detection surface area of an individual CMUT is approximately 20 $\mu \mathrm{m}$ in diameter [1-3]. Initially, CMUTs were developed for medical imaging and therapeutic purposes [4-6]. CMUTs gained attention for medical ultrasonic imaging application and significant improvements in CMUTs performance have been demonstrated during the past decade. Less attention has focused on another aspect of this technology, which is its use as a mass-sensitive sensor. Since the CMUT is a high frequency resonator, its oscillation and motion will be affected by mass change due to adsorption and desorption on its surface. For a cantilever resonator, its frequency is given by:

$$
\frac{\Delta \mathrm{f}}{\mathrm{f}}=-\frac{1}{2} \frac{\Delta \mathrm{m}}{\mathrm{m}}
$$

Where $\Delta \mathrm{f}$ is the change in resonant frequency $\mathrm{f}$ and $\Delta \mathrm{m}$ is the change in mass of the resonator with mass $\mathrm{m}$. For CMUTs with surface area A, the relationship between frequency and mass is,

$$
\frac{\Delta \mathrm{f}}{\mathrm{f}}=-\frac{1}{2} \frac{\Delta \mathrm{m}}{\mathrm{m}}
$$

The decrease in resonant frequency from its original value provides a measurement of the mass change in real time. This concept has been widely used in chemical sensing on many different types of resonators such as SAW [7], QCM [8] and micro-fabricated cantilever devices $[9,10]$. CMUTs with high resonant frequency can theoretically detect the mass of analytes in femtogram range or lower and with appropriate functionalization they can target specific analytes [1]. In the past, CMUTs were applied to detect low concentration chemical vapor such as dimethyl methylphosphonate (DMMP), decane, alcohol and acetone etc. $[1,3,11]$, however their capabilities as biosensors have not been widely explored.
Coating CMUTs with biocompatible materials such as parylene-c and polydimethylsiloxane (PDMS) [12] is achievable and useful in biological detection applications. However, it is only until recently that CMUTs were reported as a biosensor using a label-free detection scheme to observe the binding of antibodies to bovine leukemia virus protein gp51 (anti-gp51) [13] on previously gp51-coated CMUTs. In this communication, whole blood samples from cattle were used as a source of anti-gp51 and the measurements were done on CMUTs with a resonant frequency of $12 \mathrm{MHz}$. Current demands for cost savings in diagnostics and biomedical detection call for the quantitative detection of human disease markers at low concentrations $(\mathrm{pg} / \mathrm{ml} \mathrm{or} \mathrm{ng} / \mathrm{ml}$ ). These demands can at least partially be fulfilled by label-free detection technology [14]. Conventional label-dependent detection systems (e.g. Enzyme-Linked Immunosorbent Assay (ELISA)) are often expensive and time-consuming to perform and offer other challenges such as multiple label issues [15]. Therefore, development of sensitive, reliable, high-throughput-capable, label-free detection techniques is now attracting significant attention. These label-free detection techniques can be used for monitoring biomolecular interactions and simplify bioassays by eliminating the need for secondary reactants such as fluorescent, chemiluminescent or radioactive tags.

In this study, we used a CMUT-based detection system using CMUT mass-sensitive sensors with a silicon membrane surface that have a resonant frequency of $50-52 \mathrm{MHz}$, to achieve high sensitivity in protein detection. To demonstrate the potential of CMUTs for

*Corresponding author: James Gimzewski, Principal Investigator \& Satellites Co-Director, WPI Center for Materials NanoArchitectonics (MANA), NIMS, Japan E-mail: gimzewski@cnsi.ucla.edu

Received May 23, 2012; Accepted June 23, 2012; Published June 28, 2012

Citation: Eaimkhong S, Steiert M, Harper TF, Cable MD, Gimzewski J (2012) Label-Free Biodetection Using Capacitive Micromachined Ultrasonic Transducers (CMUTs) and Its Application for Cardiovascular Disease Diagnostics. J Nanomed Nanotechol 3:144. doi:10.4172/2157-7439.1000144

Copyright: (C) 2012 Eaimkhong S, et al. This is an open-access article distributed under the terms of the Creative Commons Attribution License, which permits unrestricted use, distribution, and reproduction in any medium, provided the original author and source are credited. 
Citation: Eaimkhong S, Steiert M, Harper TF, Cable MD, Gimzewski J (2012) Label-Free Biodetection Using Capacitive Micromachined Ultrasonic Transducers (CMUTs) and Its Application for Cardiovascular Disease Diagnostics. J Nanomed Nanotechol 3:144. doi:10.4172/21577439.1000144

Page 2 of 6

biosensing, we employed this technology in detecting biomolecules by testing two different schemes. The first scheme was designed to detect antibodies with the CMUT sensor. This is because when antigens infect the human body, several types of cells work together to recognize them and respond in producing excess amounts of antibodies to maintain normal regulation. Detection of antibody in plasma will imply that our body may be infected by the specific antigen to that antibody even if the antigen itself is not detectable.

Detecting antigens remains important to confirm precision and accuracy in diagnostics, i.e. disease state and progress. Consequently, we also performed an antigen detection assay as a part of the experiments. For antigen detection, we were interested in Cardiovascular Diseases (CVD) because they are the leading cause of mortality in the United States [16]. A rapid and accurate diagnosis of CVD is therefore critically important [17]. One common method to diagnose CVD is by detecting biomarkers known to have association with CVD such as C-reactive protein (CRP), Tumor Necrosis Factor (TNF- $\alpha$ ) and Interleukin 6 (IL6) [17-21] by performing an immunological assay. A similar type of test can be performed on a single CMUT chip in a short period of time without requiring multistep protocols such as fluorescently labeled secondary antibodies required for microarray or ELISA. Since much research has suggested a stronger association between TNF- $\alpha$ and CVD than most other biomarkers $[19,21]$, we decided to focus on detection of TNF- $\alpha$.

To perform an immunological assay, antibodies to capture antigens of interest have to be immobilized on the CMUTs surface so that the Fab domains are exposed [14]. We compared two methods for immobilization of antibodies. Both strategies aimed at binding the Fc domain of the antibody such that it is oriented towards the surface causing the Fab domains to be exposed to the specific antigen. For the first detection scheme, we used protein A that readily adsorbs onto silicon surfaces to immobilize human IgG onto the sensor in the desired orientation [22-24]. The second detection scheme utilized the non-specific affinity of bovine serum albumin (BSA) on silicon surface [25-27]. Biotinylated BSA functionalized sensors were therefore fabricated. This was followed by attachment of streptavidin, which provided additional biotin binding sites for subsequent capture of biotinylated antibodies.

We performed a calibration test using biotinylated Quantum Dots (Q-dots) that were captured using the same immobilization scheme as described above. After Q-dots were applied, the surface roughness parameter $\mathrm{R}_{\mathrm{a}}$ was measured from an interferometer to provide a subsequent independent measurement confirming correlation between layer thickness and the mass of the protein layers on the CMUTs (Figure 1 and Figure 2).

\section{Material and Methods}

\section{CMUT sensor, data acquisition and frequency measurements}

The CMUT data acquisition platform with built-in temperature control (developed by Matrix Sensors Inc.), operates at a high frequency of $\sim 20-60 \mathrm{MHz}$ which is in the range of the resonant frequency of current devices (50-52 MHz). This platform can measure the resonant frequency and performs a frequency sweep in less than 1s. In this setup, a single 16 channel-CMUT chip was wire-bonded onto a PCB connector, which allows plug-and-play read-out with the acquisition unit. This allows for multiple assays or chemistries to be performed simultaneously on different sensors of the same chip or multiple chips by easily swapping out CMUT chips on the reader platform. Furthermore, the compact size of the chip, only $\sim 10 \mu$ l of liquid sample required for functionalization of the entire chip surface, or less than 1 $\mu \mathrm{l}$ to functionalize individual sensors on a chip.

The electronics measure the resonant frequency of the CMUT device which decreases in response to the mass adsorbed onto the top of CMUT surface. This drop is converted to the mass adsorbed on the surface as illustrated in Figure 3.

Before frequency measurement can be performed, CMUT surface has to be dried after application of protein solution to avoid damping and reduce error in frequency reading. After the surface is dried, frequency sweep was repeated until the frequency reading stops to fluctuate (usually around $15 \mathrm{~min}$ ) and the average of the last 100 cycles was used as the resonant frequency. The surface temperature of CMUT chip was set to be a constant $35^{\circ} \mathrm{C}$.

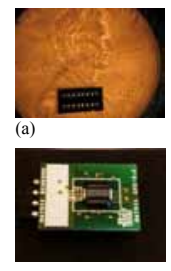

(b)

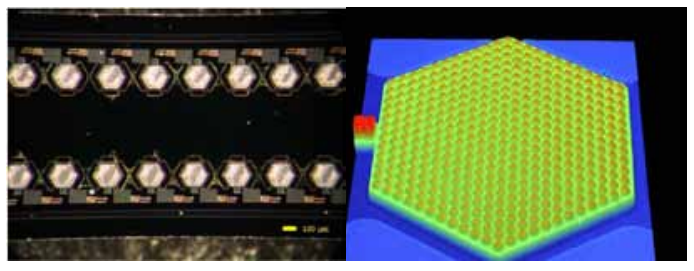

(c) (d)
Figure 1: (a) wafer-fabricated CMUT chip (approx. $2 \times 5 \mathrm{~mm}$ ) before mounting and wire-bonding; (b) wire-bonded CMUTs chip mounted to an electronic carrier board; (c) array of 16 sensors on a CMUT chip; (d) surface opticalprofiler view of a single sensor.

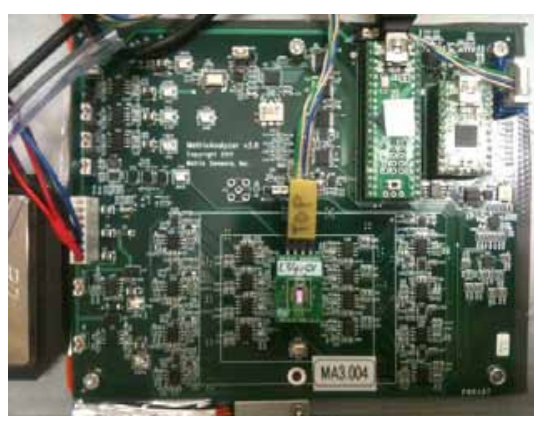

(a)

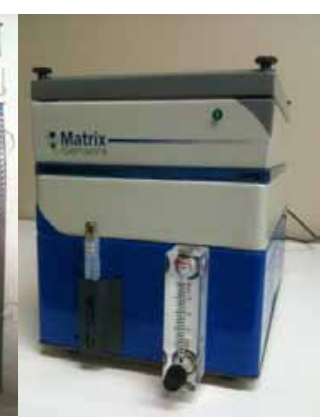

(b)
Figure 2: Data acquisition unit (a) data acquisition circuit board (b) Matrix Sensor reader that include a climate control unit (bottom part) for precise control of temperature and relative humidity inside the measurement chamber (top part).

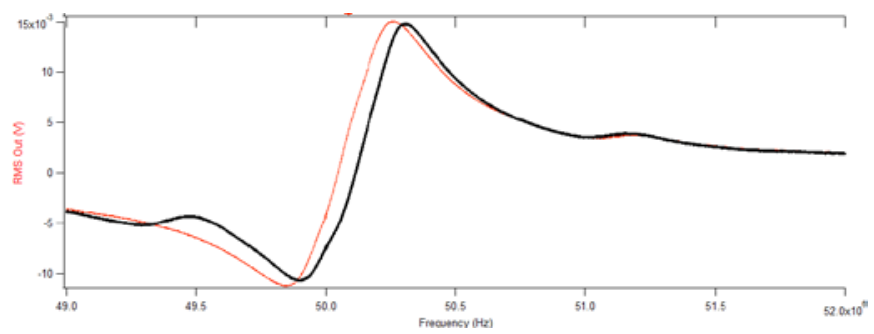

Figure 3: Frequency sweeps of an antibody-functionalized CMUT sensorbefore capture of antigen (black curve) and after binding of antigen (red curve) The resonant frequency decreased by $80 \mathrm{kHz}$ upon capture of antigen onto the antibody-functionalized sensor surface corresponding to a mass $300 \mathrm{pg}$ of antigen/sensor. 
Citation: Eaimkhong S, Steiert M, Harper TF, Cable MD, Gimzewski J (2012) Label-Free Biodetection Using Capacitive Micromachined Ultrasonic Transducers (CMUTs) and Its Application for Cardiovascular Disease Diagnostics. J Nanomed Nanotechol 3:144. doi:10.4172/21577439.1000144

\section{Calibration of mass-detection using Quantum Dots}

Before analytical detection at low concentration, CMUTs were calibrated for precision in mass detection using biotinylated Quantum Dots $(655 \mathrm{~nm})$ (Invitrogen). For the assay, BSA-LC-biotin conjugate (ImmunoPure Biotinylated BSA, Thermo Scientific) in Phosphate Buffer Saline (PBS) pH 7.4 was incubated over the chip surface at 200 $\mu \mathrm{g} / \mathrm{ml}$ for 30 minutes at room temperature. The surface was then gently rinsed with PBS containing $0.05 \%$ Tween-20 (PBS-T) followed by a rinse with DI water and air drying prior to measurement. Streptavidin (Thermo Scientific) in PBS pH 7.4 was then incubated over the BSALC-biotin coated chip surface for $30 \mathrm{~min}$ at room temperature. Rinsing, drying and measurement were repeated as described above. After that Q-Dots-biotin were incubated over the chip in concentrations ranging from $0.1-1000 \mathrm{nM}$. After the washing and drying steps were completed, the frequency difference between this step and the previous one provided the mass of Q-Dots attached onto CMUTs surface. To have a better understanding of the correlation of mass to frequency shift, the surface amplitude parameter $\left(R_{a}\right)$ of the CMUTs surface were also recorded.

\section{Antibody detection: human IgG and anti-human IgG}

Overview of detection scheme: In this experiment, we showed that immunological assays could be performed on the CMUTs without fluorescent tags. We chose a robust model of human IgG (Sigma Aldrich I4506) and anti-human IgG Fab-specific (Sigma Aldrich I9010) as the specific pair for immunological recognition while the anti-goat IgG (Sigma Aldrich A4187) served as the non-specific antigen.

To avoid denaturation of the proteins from drying out for intermittent frequency measurements, measurements were obtained only for the endpoint of the assays instead of after every functionalization step. We divided CMUTs chip into 3 sets providing 3 different measurements. The first set recorded the change in frequency of the device after being coated with protein A (Sigma Aldrich 82493), human IgG and bovine serum albumin $\left(\Delta \mathrm{f}_{0}\right)$. This was used as an arbitrary zero baseline before addition of the two antibodies. The second set measured the change in frequency of the device after being coated with protein A, human IgG, bovine serum albumin and antihuman $\operatorname{IgG}\left(\Delta \mathrm{f}_{\text {specific }}\right)$, which served as a positive control. The final value recorded was the change in frequency of a device after being coated with protein $\mathrm{A}$, human IgG, bovine serum albumin and anti-goat IgG $\left(\Delta \mathrm{f}_{\text {non-specific }}\right)$, which served as a negative control.

After all three frequency changes were obtained, the difference between $\Delta \mathrm{f}_{\text {specific }}$ and $\Delta \mathrm{f}_{0}$ gave the frequency change corresponding to the mass of anti-human IgG Fab-specific of the specific antibodies bound. Similarly the difference between $\Delta \mathrm{f}_{\text {specific }}$ and $\Delta \mathrm{f}_{0}$ gave the frequency change corresponding to the mass of anti-goat IgG or the non-specific antibodies bound.

The assays were performed with antibody concentration ranging from $10 \mathrm{ng} / \mathrm{ml}-500 \mathrm{ng} / \mathrm{ml}$ under the same condition to minimize variation in protein performance.

Functionalization: Before protein functionalization, the surface of a CMUT device was cleaned using oxygen plasma for 2 minutes and then rinsed with ethanol. The chip surface was incubated in ethanol for 10 minutes to further increase surface hydrophilicity before its baseline frequency was recorded. The functionalization began with the addition of protein A (Sigma Aldrich 82493) at $500 \mu \mathrm{g} / \mathrm{ml}$ in PBS $(\mathrm{pH}=7.4)$ and incubation for 2 hours. The surface was then washed and dried as previously described before $500 \mu \mathrm{g} / \mathrm{ml}$ of human IgG (Sigma Aldrich
I4506) was applied and incubated for 2 hours. Washing and drying were repeated and, as before, $0.1 \%$ Bovine Serum Albumin (BSA) was added and incubated for 30 minutes (blocking step). After washing and drying, some of the chips were tested for frequency. The others were separated into two sets for positive and negative controls. For positive control or specific binding, anti-human IgG Fab-specific, at concentrations of $10 \mathrm{ng} / \mathrm{ml}-500 \mathrm{ng} / \mathrm{ml}$ in $0.02 \%$ BSA in PBS, was added and incubated for 3 hours. Washing and drying was again repeated before frequency measurements. Similarly for negative control or non-specific binding, anti-human IgG Fab-specific at concentrations of $10 \mathrm{ng} / \mathrm{ml}-500 \mathrm{ng} /$ $\mathrm{ml}$ in $0.02 \%$ BSA in PBS was added and incubated for 3 hours. Washing and drying was repeated before the frequency measurements.

\section{Quantitative TNF-a detection on a single CMUTs chip}

We performed the assay to detect TNF- $\alpha$ on CMUTs system by immobilization of anti- TNF- $\alpha$ on the surface using biotin/streptavidin binding. The assay started by applying BSA-LC-biotin conjugate (ImmunoPure biotinylated BSA, Thermo Scientific) in PBS pH 7.4, which was incubated over the chip surface at $200 \mu \mathrm{g} / \mathrm{ml}$ for 30 minutes at room temperature. The surface was then gently rinsed with Phosphate Buffer Saline with $0.05 \%$ Tween-20 (PBS-T) followed by a rinse with DI water and air drying before measurement. Streptavidin (Thermo Scientific) in PBS pH 7.4 was then incubated over the BSA-LC-biotin coated chip surface for $30 \mathrm{~min}$ at room temperature. Rinsing, drying and measurement were repeated as described previously. After that $0.5 \mu \mathrm{g} / \mathrm{ml}$ of anti-TNF- $\alpha$-biotin conjugate (anti-human TNF-alpha, biotinylated, affinity-purified PAB from R\&D systems) were incubated on the chip. After the washing and drying steps were completed, TNF- $\alpha$ antigens at different concentrations, over the range from $0-400 \mathrm{ng} / \mathrm{ml}$ were applied onto the previously coated chips. The adsorbed mass of TNF- $\alpha$ from each concentration was determined from the frequency difference between this and the previous step. A calibration curve of TNF- $\alpha$ detection over the concentration was then created.

\section{Results and Discussions}

\section{Sensitivity and reliability of CMUT sensor for detection of biomolecular interaction}

A mass-sensing calibration of the CMUTs for the detection of biomolecular interaction was conducted by applying a layer of Q-dots (Invitrogen) onto the device utilizing the surface chemistry described in the manufacturer's kit. Over a concentration range of $0.01-1,000 \mathrm{nM}$, a calibration curve of the frequency drop (frequency decrease) from an average of 8 sensors per CMUT chip over this range of concentration was obtained (Figure 4).

In order to verify the reliability of the frequency drop as a good representation of the protein mass adsorbed on top of the device, the coated devices were measured by optical surface interferometry (Bruker NT9300) to determine the vertical surface roughness parameter $\left(R_{a}\right)$. This value represents the thickness of the deposited layer on a surface from the average surface value and is a good indicator for the amount of material deposited on the surface. The $\mathrm{R}_{\mathrm{a}}$ values of the coated devices were plotted against the Q-dot concentration and the calibration curve shows a reasonable linear relationship similarly to the graph obtained from the measurements of frequency change (frequency drop). The frequency drop is correlated with $\mathrm{R}$ thickness with a correlation efficient of 0.90 . This means that the mass calculated from frequency drop can be used in biological mass sensing to detect the presence of a particular protein using appropriate coating techniques on CMUTs. Within the standard deviations, the plots in Figure 4 exhibit a reasonable linearity. 
Citation: Eaimkhong S, Steiert M, Harper TF, Cable MD, Gimzewski J (2012) Label-Free Biodetection Using Capacitive Micromachined Ultrasonic Transducers (CMUTs) and Its Application for Cardiovascular Disease Diagnostics. J Nanomed Nanotechol 3:144. doi:10.4172/21577439.1000144

A slight drop at $1 \mathrm{nM}$ over $0.01 \mathrm{nM}$ could be due to lack of precision in sample preparation and some variation in the volume-to-surface ratio. Alternatively this may also indicate that the lower limits of detection for the particular surface functionalization protocol chosen had been reached - and in fact the presented curve would hence represent the lower concentration range of a sigmoidal dose-dependency curve. It should be noted that further experimental investigations are underway promulgated by these initial findings to fully elucidate the label-free antibody binding studies of this novel methodology in relation to currently used technologies.

\section{Antibody detection on CMUT sensor}

As mentioned earlier, detection of antibodies are important and is an indirect detection method for disease-causing agents in our body. We chose to immobilize human IgG at its Fc position using protein A and hence the Fab domains of this antibody are exposed and available for antigen binding. When the anti-human IgG Fab specific was added, the Fab units of anti-human IgG should readily bind to the Fab units of previously coated human IgG. This interaction is strong enough in order for the specific antibody to remains on the device even after many washes. Unlike ELISA, our technique does not rely on secondary fluorescently labeled antibodies, where the signal from the fluorescent tags can be overestimated resulting in higher than present analyte concentrations [15]. The signal obtained from CMUT mass-sensitive devices is the direct representation of mass of the bound antibody bound and does not suffer from amplification effects by secondary antibody tags.

To assure that the mass derived from frequency difference in the specific antibody assay is primarily from the binding event of a specific antibody i.e. to ensure the observed signal is from the protein of interest, it is crucial to be able to clearly resolve between the mass of specific and non-specific antibody at the same concentrations. Both masses were compared in Figure 5 after both specific and nonspecific antibody assays were performed. For accuracy and reliability of the data, all assays were performed at the same time and under the same conditions in moist chambers to avoid variation in the protein performance and dehydration of the protein during surface incubation. The results below clearly show significant difference in mass between specific and non-specific antibodies bound to the surface for 4 antibody concentrations. Ideally, we expect the mass contributed by non-specific binding events to be negligible; however, it is typical that some crossbinding of non-specific proteins or antibodies to the human IgG occurs (Figure 5).

In this method, CMUTs are able to resolve the mass between specific and non-specific down to $10 \mathrm{ng} / \mathrm{ml}$. To a certain extent, biodetection using CMUTs technology can still be optimized to work at much lower protein concentrations which we expect to be in the $\mathrm{pg} / \mathrm{ml}$ range. The main limitation of CMUTs technology for protein detection is the level of electrical noise that affects the detection at very low amounts of protein concentration.

\section{Quantitative TNF- $\alpha$ detection and CMUTs potential for disease diagnostics}

In cardiovascular diseases, acute symptoms such as stroke or heart attack can arise in patients shown to have high levels of TNF- $\alpha$ in their blood. Hence, sensitive, rapid, and inexpensive detection methods using CMUT mass-sensitive sensors could benefit patients with cardiovascular disease risk to monitor their condition more efficiently.

In medical diagnostics, a TNF- $\alpha$ level of greater than $10 \mathrm{pg} / \mathrm{ml}$ in the blood indicates an increased risk of cardiovascular diseases [21]. In this study, we therefore focused on observing the sensitivity of TNF- $\alpha$ detection in comparison to this critical value to evaluate the potential of our technology for cardiovascular diseases diagnostics.

A slightly different immobilization technique was employed by applying BSA to initiate protein functionalization on the CMUTs. The BSA-biotin conjugate was used as the first layer to allow streptavidin to bind onto the surface. After that biotinylated anti-TNF- $\alpha$ (binding at Fc domain) was added respectively and the streptavidin/biotin interaction assists immobilization of the antibodies so that the Fab regions of antiTNF- $\alpha$ are exposed at the interface. It is noted that a small fraction of biotinylated anti-TNF- $\alpha$ can immobilize Fab domains of TNF- $\alpha$ on the surface instead of $\mathrm{Fc}$ domains.

TNF- $\alpha$ at clinically relevant concentrations over the range of 0 $400 \mathrm{ng} / \mathrm{ml}$ was then applied on anti-TNF- $\alpha$-coated devices and the calibration curve shows an excellent linear dependency of frequency shift as a function of concentration of TNF- $\alpha$. This quantitative analysis

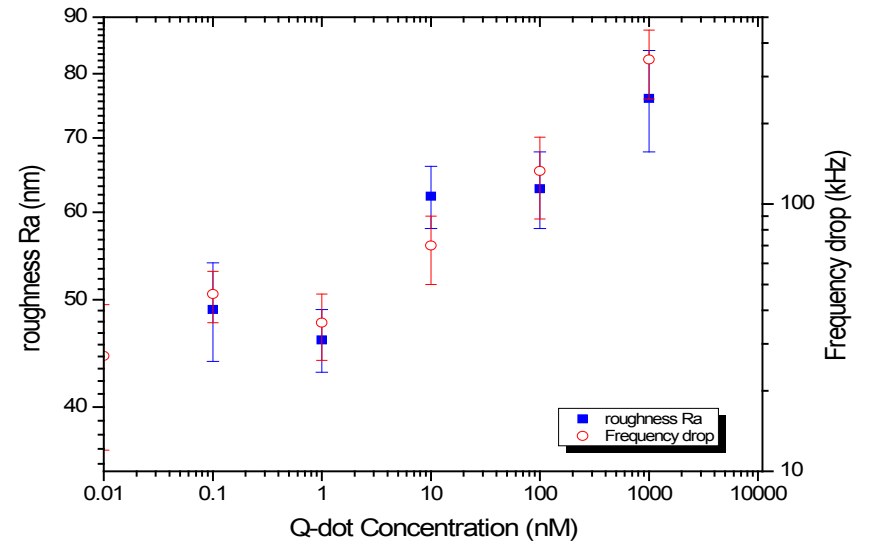

Figure 4: Calibration curve from frequency change corresponded to addition of $Q$-dot solution on CMUTs surface (open circle) and calibration curve from $R_{a}$ surface roughness of the coated CMUTs chips (solid square).

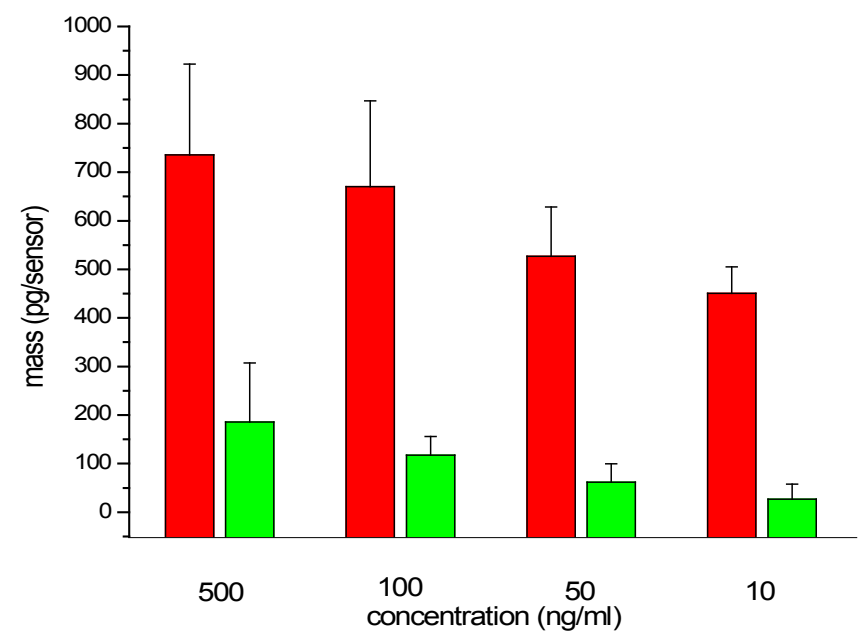

Figure 5: Specific (red) and non-specific (green) antibody mass bound at the concentration of $10 \mathrm{ng} / \mathrm{ml}-500 \mathrm{ng} / \mathrm{ml}$ ( $n=8$, out of 2 CMUT chips). The detection sensitivity is $0.0035 \mathrm{pg} / \mathrm{Hz}$ sensor. 
Citation: Eaimkhong S, Steiert M, Harper TF, Cable MD, Gimzewski J (2012) Label-Free Biodetection Using Capacitive Micromachined Ultrasonic Transducers (CMUTs) and Its Application for Cardiovascular Disease Diagnostics. J Nanomed Nanotechol 3:144. doi:10.4172/21577439.1000144

Page 5 of 6

can be used to determine the concentration of TNF- $\alpha$ and is a viable method for this protein over the critical range (Figure 6).

Although this calibration shows an excellent linearity $\left(\mathrm{R}^{2}=0.99\right)$ that suggests reliability of detection of TNF- $\alpha$ on CMUT devices, it would be interesting to extend the range of concentrations to include concentrations between $1-10 \mathrm{ng} / \mathrm{ml}$ which is the clinically interesting concentration range that would allow making the decision regarding cardiovascular risk (i.e. the $10 \mathrm{ng} / \mathrm{ml}$ cut-off point). The inclusion of additional cardiovascular disease markers alongside with TNF- $\alpha$ i.e. CRP and IL-6 to create a cardiovascular panel on a single CMUT chip should provide a promising approach to a more comprehensive cardiovascular disease diagnostic tool in the future.

\section{Conclusions}

In this study, we performed different functionalization schemes on $\mu \mathrm{m}$-sized biocompatible CMUTs demonstrating its potential to perform biodetection of specific proteins with accuracy and high sensitivity. In contrast to ELISA, for antigen detection assays, antigen capture alone is sufficient for detection, i.e. no labeling and secondary antibodies (for signal and amplification) are required. The antigen is introduced and applied as the final step of the assay onto the sensor chip. This means that the sensor chips can be pre-fabricated as singlestep kits, which is very cost-effective and time saving for research and diagnostic applications.

Furthermore, the size of the substrate is very small and the presented method only requires a minimal volume of $10 \mathrm{ul}$ to cover the entire 16-sensor chip without. This contributes to an additional cost reduction by requiring fewer quantities of reagent materials.

In our laboratory, CMUTs are being developed to be fully handheld units relying only on battery power or power via a USB connection from a laptop computer and being operated either as a stand-alone unit or via a computer interface. This will facilitate the use for diagnostic applications in medically underserved and hard-to-reach regions or for soldiers in the battle fields avoiding the need to ship samples to a central facility of laboratory for analysis.

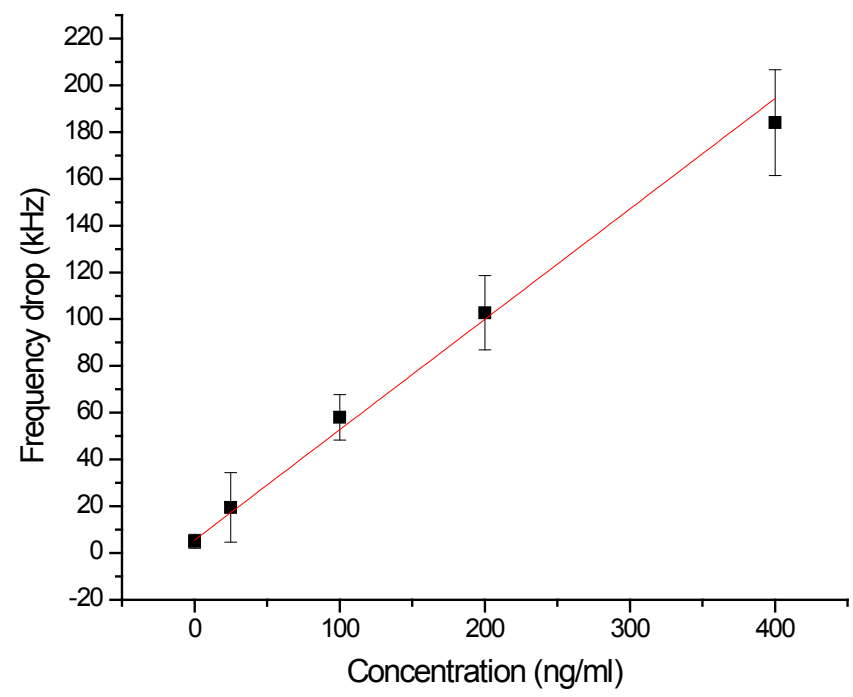

Figure 6: Calibration curve of frequency changes in correspond to concentration of TNF- $\alpha$.
With current progress underway, this technology may eventually be capable of exceeding the detection limits of ELISA even though as of today this novel detection method still has a lower sensitivity compared to ELISA. Despite this fact, it is already interesting for certain diagnostics applications, an analyte concentration in the range of 10 $1,000 \mathrm{ng} / \mathrm{ml}$ in the blood of the patient is clinically relevant for a variety of disease markers (including TNF- $\alpha$ ) and can be easily performed with this new technology. Additionally, some of the advantages mentioned above such as faster detection time, lower cost and the small amounts of materials required as well as its portability and ability for further miniaturization of this technology make CMUTs an interesting alternative for protein detection in a laboratory or medical setting.

\section{Acknowledgement}

This work was supported by Matrix Sensors Inc. The author would like to thanks Brandon Burbach, an undergraduate student for his ample assistance on interferometry.

\section{References}

1. Lee HJ, Park KK, Kupnik M, Oralkan Ö, Khuri-Yakub BT (2011) Chemical vapor detection using a capacitive micromachined ultrasonic transducer. Anal Chem 83: 9314-9320.

2. Lee HJ, Park KK, Cristman P, Oralkan Ö, Kupnik M, et al. (2009) A low-noise oscillator based on a multi-membrane CMUT for high sensitivity resonant chemical sensors. 22th IEEE MEMS Conference, Sorrento, Italy.

3. Park KK, Lee H, Kupnik M, Oralkan Ö, Ramseyer JP, et al. (2011) Capacitive micromachined ultrasonic transducer (CMUT) as a chemical sensor for DMMP detection. Sens Actuators B: Chem 20: 1120-1127.

4. Wong SH, Watkins RD, Kupnik M, Butts-Pauly K, Khuri-Yakub BT (2008) Feasibility of MR-Temperature Mapping of Ultrasonic Heating from a CMUT. IEEE Trans Ultrason Ferroelectr Freq Control 55: 811-818.

5. Khuri-Yakub BT, Oralkan O (2011) Capacitive micromachined ultrasonic transducers for medical imaging and therapy. J Micromech Microeng 21 54004-54014

6. Wong SH, Kupnik M, Watkins RD, Butts-Pauly K, Khuri-Yakub BT (2010) Capacitive Micromachined Ultrasonic Transducers for Therapeutic Ultrasound Applications. IEEE Trans Biomed Eng 57: 114-123.

7. Snow A, Wohltjen H (1984) Poly(ethylene maleate)-cyclopentadiene: a mode reactive polymer-vapor system for evaluation of a SAW(Surface Acoustic Wave) microsensor. Anal Chem 56: 1411-1416.

8. Bruckenstein S, Shay M (1985) Experimental aspects of use of the quartz crystal microbalance in solution. Electrochim Acta 30: 1295-1300.

9. Battiston FM, Ramseyer JP, Lang HP, Baller MK, Gerber CH, et al. (2001) A chemical sensor based on a microfabricated cantilever array with simultaneous resonance-frequency and bending readout. Sens Actuators B: Chem 77: 122 131

10. Wali RP, Wilkinson PR, Eaimkhong S, Hernando-Garciaa J, Sánchez-Rojasd JS, et al. (2010) Fourier transform mechanical spectroscopy of micro-fabricated electromechanical resonators: A novel, information-rich pulse method for sensor applications. Sens Actuators B: Chem 147: 508-516.

11. Park KK, Lee HJ, Yaralioglu GG, Ergun AS, Oralkan O, et al. (2007) Capacitive micromachined ultrasonic transducers for chemical detection in nitrogen. App Phys Lett 91: 094102.

12. Zhuang X, Nikoozadeh A, Beasley MA, Yaralioglu GG, Khuri-Yakub BT, et al. (2007) Biocompatible coatings for CMUTs in a harsh, aqueous environment. J Micromech Microeng 17: 994-1001.

13. Ramanaviciene A, Virzonis D, Vanagase G, Ramanavicius A (2010) Capacitive micromachined ultrasound transducer (CMUT) for immunosensor design. Analyst 135: 1531-1534.

14. Morrison D W G, Dokmeci M R, Demirci U, Khademhosseini A (2008) Clinica applications of micro- and nanoscale biosensors. John Wiley \& Sons, USA.

15. Ray S, Mehta G, Srivastava S (2010) Label-free detection techniques for protein microarrays:Prospects, merits and challenges. Proteomics 10: 731-748. 
Citation: Eaimkhong S, Steiert M, Harper TF, Cable MD, Gimzewski J (2012) Label-Free Biodetection Using Capacitive Micromachined Ultrasonic Transducers (CMUTs) and Its Application for Cardiovascular Disease Diagnostics. J Nanomed Nanotechol 3:144. doi:10.4172/21577439.1000144

16. Thom T, Haase N, Rosamond W, Howard VJ, Rumsfeld J, et al. (2006) Heart disease and stroke statistics--2006 update: a report from the American heart association statistics committee and stroke statistics subcommittee. Circulation 113: e85-151.

17. Tang L, Ren Y, Hong B, Kang KA (2006) Fluorophore-mediated, fiber-optic multi-analyte,immunosensing system for rapid diagnosis and prognosis of cardiovascular diseases. J Biomed Opt 11: 021011.

18. Sabatine MS, Morrow DA, de Lemos JA, Gibson CM, Murphy SA, et al. (2002) Multimarker approach to risk stratification in non-ST elevation acute coronary syndromes: simultaneous assessment of troponin I, C-reactive protein, and B-type natriuretic peptide. Circulation 105: 1760-1763.

19. Pai JK, Pischon T, Ma J, Manson JE, Hankinson SE, et al. (2004) Inflammatory markers and the risk of coronary heart disease in men and women. $\mathrm{N}$ Engl $\mathrm{J}$ Med 351: 2599-2610.

20. Tuomisto K, Jousilahti $P$, Sundvall J, Pajunen P, Salomaa V (2006) C-reactive protein, interleukin-6 and tumor necrosis factor alpha as predictors of inciden coronary and cardiovascular events and total mortality. A population-based, prospective study. Thromb Haemost 95: 511-518.

21. Cesari M, Penninx BW, Newman AB, Kritchevsky SB, Nicklas BJ, et al. (2003)
Inflammatory Markers and Cardiovascular Disease (The Health, Aging and Body Composition [Health ABC] Study). Am J Cardiol 92: 522-528.

22. Wang Z, Jin G (2003) Feasibility of protein A for the oriented immobilization of immunoglobulin on silicon surface for a biosensor with imaging ellipsometry. J Biochem Biophys Methods 57: 203-211.

23. Dutra RF, Castro CM, Azevedo CR, Vinhas E, Malagueño E, et al. (2000) Immobilization of pneumococcal polysaccharide vaccine on silicon oxide wafer for an acoustical biosensor. Biosens Bioelectron 15: 511-514.

24. Ikeda T, Hata Y, Ninomiya K, Ikura Y, Takeguchi K, et al. (2009) Oriented immobilization of antibodies on a silicon wafer using Si-tagged protein A. Anal Biochem 385: 132-137.

25. McClellan SJ, Franses El (2005) Adsorption of bovine serum albumin at solid/ aqueous interfaces. Colloids Surf A: Physicochem Eng Asp 260: 265-275.

26. Cummings $L$ (2010) Characterization of Biomolecular Interactions at the Silicon Photonics Interface. NNIN REU Research Accomplishments, Washington, USA.

27. Lee CS, Lee SH, Park SS, Kim YK, Kim BG (2003) Protein patterning on siliconbased surface using background hydrophobic thin film. Biosens Bioelectron 18 437-444. 